\title{
A note on 1-planar graphs with minimum degree 7
}

\author{
Therese Biedl * \\ David R. Cheriton School of Computer Science, University of Waterloo, Waterloo, \\ Ontario N2L 1A2, Canada. biedl@uwaterloo.ca
}

\begin{abstract}
It is well-known that 1-planar graphs have minimum degree at most 7 , and not hard to see that some 1-planar graphs have minimum degree exactly 7 . In this note we show that any such 1-planar graph has at least 24 vertices, and this is tight.
\end{abstract}

\section{Introduction}

A 1-planar graph is a graph that can be drawn in the plane such that every edge has at most one crossing. (We refer the reader to any graph theory textbook for standard notations around graphs.) This graph class was introduced by Ringel [5], because they naturally arise when taking a planar graph $G$ and its dual graph $G^{*}$ and connecting vertices/faces that are incident to each other. The resulting 1-planar graph $H$ has a vertex of degree at most 6 , because at least one of $G$ and $G^{*}$ has a vertex $v$ of degree 3 , and adding the edges to obtain $H$ doubles the degrees.

However, not all 1-planar graphs are obtained by combining a planar graph and its dual; for example $K_{6}$ is 1-planar but not obtained in this way. A different way to argue this is to exhibit a 1-planar graph that has no vertex of degree 6 or less. Figure 1 shows one such graph; it has 24 vertices. In this note, we argue that "24" is tight. Many other structural properties are known for 1-planar graphs (see for example [2]3), and even some for 1-planar graphs of minimum degree 7 4, but it seems that the size of 1-planar graphs with minimum degree 7 has not previously been studied.

\section{Lower bound}

Let $G$ be a simple 1-planar graph with $n$ vertices, $m$ edges and minimum degree 7. We assume throughout the following argument that one particular 1-planar drawing $\Gamma$ (described by giving the clockwise order around each vertex and the information which pairs of edges are crossing) has been fixed. We assume that this drawing is good [6], which in particular means that no edge crosses itself and no two edges with a common endpoint cross.

The given 1-planar drawing defines the regions, which are the connected parts of $\mathbb{R} \backslash \Gamma$. Each region $R$ contains on its boundary a number of vertices and

\footnotetext{
* Supported by NSERC.
} 


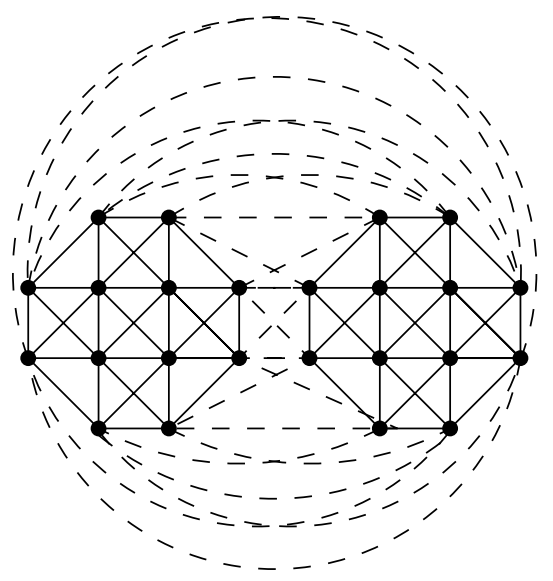

Figure 1. A 1-planar graph with minimum degree 7.

crossings of $\Gamma$; these are called the corners of $R$. The degree of a region is the number of corners that it has. No region can have one or two corners, because this would either mean a loop or a double edge in the graph, or the drawing would not have been good.

We call a 1-planar drawing triangulated if every region has exactly three corners. Note that the given 1-planar drawing $\Gamma$ can be made triangulated by inserting further edges as follows. We already know that no region has fewer than three corners. If some region $R$ has four or more corners, then we can find two non-consecutive corners $v, w$ on $R$ that are both vertices, and add an edge between them. Edge $(v, w)$ can be drawn inside $R$ without crossing, and either splits $R$ into two regions of smaller degree, or decreases the number of connected components of the boundary of $R$; either way we have made progress and can repeat until the resulting drawing is triangulated and 1-planar. Note that this triangulated 1-planar drawing may not represent a simple graph, but in what follows simplicity will never be used, only that the drawing is triangulated.

So fix from now on a triangulated 1-planar drawing $\Gamma$ where all vertices have degree 7 or more. Let $n$ and $m$ be the number of edges in the graph that $\Gamma$ represents; note that $n \geq 3$ since the drawing is good and triangulated. Let $n_{7}$ be the number of vertices that have degree exactly 7 . Since all other vertices have degree at least 8 , we have

$$
2 m=\sum_{v \in V} \operatorname{deg}(v) \geq 7 n_{7}+8\left(n-n_{7}\right)=8 n-n_{7} .
$$

It is well-known that every 1-planar simple graph with $n \geq 3$ vertices has at most $4 n-8$ edges (see e.g. 3]), but we repeat the argument here because our graphs are not necessarily simple (but have a triangulated drawing) and because 
we need a more detailed bound. Let $x$ be the number of crossings of $\Gamma$. Let $\Gamma^{-}$be the planar drawing obtained by picking one edge at each of these crossings and removing it. Since $\Gamma$ was triangulated, every crossing was surrounded by four regions that are triangles, and removing one of the crossing edges hence gives two regions that are triangles. Therefore $\Gamma^{-}$is a planar triangulated drawing. It may have multiple edges, but since all regions are triangles it nevertheless follows from Euler's formula that $\Gamma^{-}$has $3 n-6$ edges. Therefore

$$
m=3 n-6+x .
$$

Let $t$ be the number of regions of $\Gamma$ that are uncrossed, i.e., for which none of the edges are crossed. Put differently, $t$ counts the number of regions where all three corners are vertices. Returning to the planar triangulated drawing $\Gamma^{-}$, observe that these $t$ regions of $\Gamma$ are also regions of $\Gamma^{-}$, and $\Gamma^{-}$has a further $2 x$ regions that correspond to the $x$ crossings of $\Gamma$. This covers all regions of $\Gamma^{-}$. By Euler's formula the triangulated planar drawing $\Gamma^{-}$has $2 n-4$ regions, and therefore

$$
2 n-4=2 x+t
$$

(In particularly therefore $m \leq 4 n-8-\frac{t}{2}$ for any 1-planar graph with a triangulated drawing.)

Consider a vertex $v$ of degree 7 in $\Gamma$. Then no two edges $e_{1}, e_{2}$ that are incident to $v$ and consecutive in the circular clockwise order at $v$ can be crossed, else the region incident to $v$ and between $e_{1}$ and $e_{2}$ would not have degree 3 . Since the degree of $v$ is odd, therefore there are two consecutive uncrossed edges at $v$, say they are $\left(v, w_{0}\right)$ and $\left(v, w_{1}\right)$. Then $\left\{v, w_{0}, v_{1}\right\}$ is an uncrossed region since $\Gamma$ is triangulated. So for every vertex of degree 7 , there exists an incident uncrossed region. Vice versa, every uncrossed region is incident to at most three vertices of degree 7 , and so we have

$$
n_{7} \leq 3 t
$$

Now we put these equations together as follows:

$3 n_{7} \stackrel{(1)}{\geq} 24 n-6 m \stackrel{(2)}{\geq} 24 n-(18 n-36+6 x) \stackrel{(3)}{\geq} 6 n+36-(6 n-12-3 t) \stackrel{(4)}{\geq} 48+n_{7}$

and the following holds.

Theorem 1. Any simple 1-planar graph with minimum degree 7 has at least 24 vertices of degree $\%$.

In particular, $G$ cannot have fewer than 24 vertices overall. Figure 1 shows that this is tight. We also note that the result holds more broadly for any 1planar graph with minimum degree 7 that has a 1-planar drawing where all regions have degree three or more, even if the graph has multiedges or loops. 


\section{Conclusion}

In this note, we argued that any simple 1-planar graph with minimum degree 7 has at least 24 vertices. This bound is tight. Our bound has some consequences for matching-bounds. Wittnebel [7] showed that there is an infinite class of simple 1-planar graphs of minimum degree 7 for which any matching $M$ has size at most $\frac{15 n+16}{31}$. He used as ingredient a 1-planar graph with minimum degree 7 and 32 vertices. Because the graph in Figure 1 has fewer vertices, this bound can be improved.

Lemma 1. For any $N$, there exists a simple 1-planar graph with minimum degree 7 and $n \geq N$ vertices for which any matching has size at most $\frac{11 n+12}{23}$.

Proof. Let $n \geq N$ be such that $n=1 \bmod 23$. Create one vertex $v$, and split the remaining vertices into $(n-1) / 23$ groups of 23 vertices each. For each group, insert edges to turn these 23 vertices, plus vertex $v$, into the graph of Figure 1. Put differently, combine $(n-1) / 23$ copies of this graph at one of their vertices. Clearly the resulting graph is 1-planar, simple, and has minimum degree 7 . Observe that $G \backslash v$ has $(n-1) / 23$ connected components, each of odd size. The bound now follows from the "easy" direction of the Tutte-Berge theorem [1]: In any matching $M$, at most one edge of $M$ connects $v$ to one component, so there are at least $\frac{n-1}{23}-1=\frac{n-24}{23}$ vertices (one in each of the other components) that are unmatched. Therefore any matching has size at most $\frac{1}{2}\left(n-\frac{n-24}{23}\right)=\frac{11 n+12}{23}$.

Because there are no smaller 1-planar graphs with minimum degree 7 , there is some hope for showing that the bound in Lemma 1 is tight (i.e., there is a matching of size at least $\frac{11 n+12}{23}$ in any 1-planar graph with minimum degree 7), though this remains an open problem.

\section{References}

1. C. Berge. Sur le couplage maximum d'un graphe. Comptes Rendus de l'Académie des Sciences, Paris, 247:258-259, 1958.

2. Franz-Josef Brandenburg, David Eppstein, Andreas Gleißner, Michael T. Goodrich, Kathrin Hanauer, and Josef Reislhuber. On the density of maximal 1-planar graphs. In Walter Didimo and Maurizio Patrignani, editors, Graph Drawing - 20th International Symposium, GD 2012, Redmond, WA, USA, September 19-21, 2012, Revised Selected Papers, volume 7704 of Lecture Notes in Computer Science, pages 327-338. Springer, 2013.

3. Igor Fabrici and Tomáš Madaras. The structure of 1-planar graphs. Discrete Mathematics, 307(7):854 - 865, 2007.

4. Dávid Hudák and Tomáš Madaras. On local properties of 1-planar graphs with high minimum degree. Ars Mathematica Contemporana, 4(2):245-254, 2011.

5. G. Ringel. Ein Sechsfarbenproblem auf der Kugel. Abh. Math. Sem. Univ. Hamburg, 29:107-117, 1965.

6. Marcus Schaefer. Crossing Numbers of Graphs. CRC Press, 2017.

7. J. Wittnebel. Bounds on maximum matchings in 1-planar graphs. Master's thesis, University of Waterloo, January 2019. http://hdl.handle.net/10012/14445 